\title{
Transitional flow patterns behind a backstep with porous-based fluid injection
}

\author{
Go-Long Tsai ${ }^{\text {a }}$, Y.C. Lin ${ }^{\mathrm{b}}$, W.J. Ma ${ }^{\mathrm{b}}$, H.W. Wang ${ }^{\mathrm{b}}$, J.T. Yang ${ }^{\mathrm{c}, *}$ \\ ${ }^{a}$ Department of Vehicle Engineering, National Taipei University of Technology, Taipei 10600, Taiwan \\ ${ }^{\mathrm{b}}$ Department of Power Mechanical Engineering, National Tsing Hua University, No. 101, Section 2, Kuang-Fu Road, Hsinchu 30013, Taiwan \\ ' Department of Mechanical Engineering, National Taiwan University, No. 1, Section 4, Roosevelt Road, Taipei 10617, Taiwan
}

\section{A R T I C L E I N F O}

\section{Article history:}

Received 2 February 2005

Received in revised form 24 May 2008

Available online $\mathrm{xxxx}$

\section{Keywords:}

Backstep

Flow pattern

Flow visualization

LDV

\begin{abstract}
A B S T R A C T
The flow structure downstream of a backstep with mass injection from a porous base was analyzed both qualitatively and quantitatively in the transitional flow regime of $R e_{\mathrm{h}}=2009-3061$. By increasing the wall injection velocity ratio gradually, four distinct flow patterns, shifted from pattern A to B, C and D, were categorized. Pressure distributions of these patterns were dominated by the wall injection velocity ratio, and various downstream-flowing tendencies were produced correspondingly. The effect of flow stabilization by decreasing the Reynolds number became more prominent if the wall injection velocity ratio was increased. Due to the existence of a shear layer, a large value of the Reynolds stress was measured near the tip of the step in pattern A. Once the wall injection was initiated, the local strength of Reynolds stress at the same location was decreased. By increasing the wall injection velocity ratio, the region with decreased level of Reynolds stress extended gradually from the tip of backstep to the streamwise location $x=0.45 X_{\mathrm{r}}$. The turbulent kinetic energy in pattern A was mostly contributed by the horizontal fluctuation of flow near the backstep in the recirculation zone, and the region with maximum horizontal fluctuation was found to evolve toward the base as the flow moves downstream. However, the weighting of vertical fluctuation became more significant as the wall injection velocity ratio increased.
\end{abstract}

(c) 2008 Elsevier Ltd. All rights reserved.

\section{Introduction}

Transpiration or film cooling is generally achieved by injecting coolant through a porous or perforated plate to form a layer of low-temperature fluid for thermal shielding. It has been extensively utilized in various engine components and combustors, such as gas-turbine blades and liquid fueled rocket engine thrust chambers. All these applications involve the separation-reattachment flow with wall injection, which is usually investigated experimentally by establishing a sudden-expansion channel with a porous base downstream of the backstep. Several characteristics are demonstrated in this type of flow, including free stream, shear layer, recirculation zone, reattachment zone, and redeveloped boundary layer. To achieve satisfactory cooling performance, the physical mixing of fluids between the inlet mainstream and base injection is critical, which provides the motivation of the present work.

Fundamental features of the separation-reattachment flow without base injection have been investigated extensively [1-6], whereas relatively less effort has been devoted to the effects of wall injection on mixing process [7-12]. Bradshaw and Wong [2] reported that the reattachment length varied between six and eight times of the height of backstep based on various inlet condi-

\footnotetext{
* Corresponding author. Tel.: +88623366 9875; fax: +88622737 7189.

E-mail addresses: golong@ntut.edu.tw (G.-L. Tsai), jtyang@ntu.edu.tw (J.T. Yang).
}

tions. And according to de Brederode and Bradshaw [13], the average flow at the spanwise centerline plane downstream of the backstep was considered two-dimensional if the aspect ratio of the test section was larger than 10 .

The injected fluid from wall adds extra mass to the flow and alters the structure of it. Richardson et al. [7] reported that wall injection decreased the recirculating velocity in separation zone. Yang and Kuo [9] found that the velocity fluctuation and Reynolds stress of the flow were decreased by increasing the relative amount of wall injection. Harinaldi and Mizomoto [12] investigated the coherent structure of flow over a backstep with gas injection from a slot. The mean flow, turbulence properties and characteristics of the coherent structure were significantly influenced by the increase of injection momentum near the backstep. Under the condition of significant mass entrainment, the velocity, size and detachment of the coherent structure were found to be suppressed by the alteration of turbulence structure in the shear layer and hydrodynamic disturbances from injection.

While most of the previous studies focused on the effects of base injection under specific rates, the transition among various flow patterns via variation of wall injection was rarely investigated. In this work, four distinct flow patterns under various wall injection velocity ratios are experimentally categorized and analyzed. Results from this study are expected to be valuable for further development of transpiration or film cooling technologies. 


\section{Experiment design}

\subsection{Test facilities}

A sudden-expansion channel (inlet channel height $30 \mathrm{~mm}$, width $W 200 \mathrm{~mm}$, and height of backstep $H 15 \mathrm{~mm}$ ) was established as the test section. The corresponding aspect ratio (AR, ratio of channel width $W$ to step height $H$ ) was 13.3 , and the average flow downstream of the backstep at the spanwise centerline plane $(z=0$ plane) of test section satisfied the criterion of being twodimensional [13]. As shown in Fig. 1, a porous plate (width

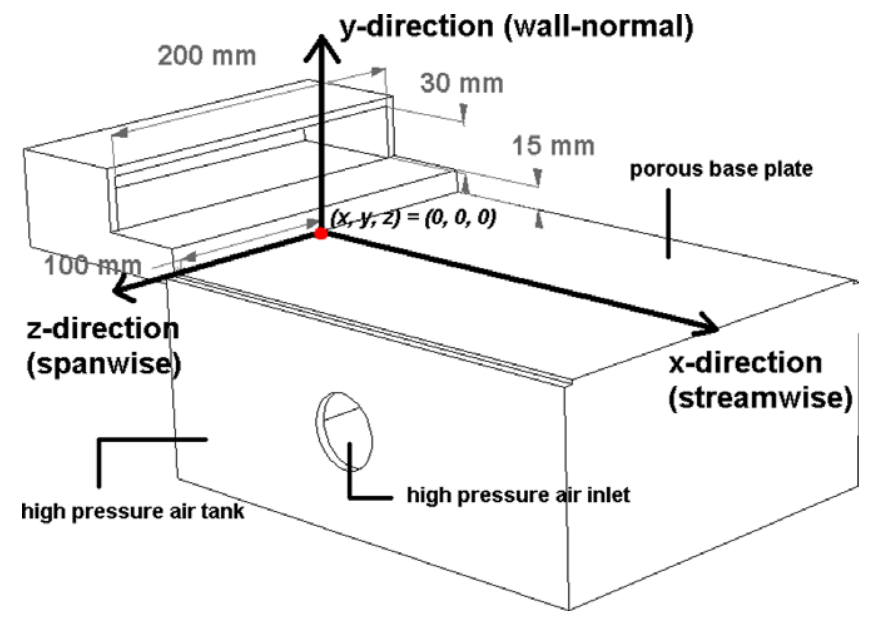

Fig. 1. Schematic diagram of the test section.
$200 \mathrm{~mm}$ and length $300 \mathrm{~mm}$ ) made of stainless steel was installed downstream of the backstep as the base. The average pore size was $10 \mu \mathrm{m}$ and the overall porosity ( $\Phi$ ) of the plate was $40.8 \%$. For simulating the flow under transpiration and film cooling conditions, air at high pressure was pumped into a settling tank connected underneath the test section first and then injected through the porous bottom wall.

A schematic diagram of the experiment setup was shown in Fig. 2. The wind-tunnel system comprised of a $76 \mathrm{~kW}$ air blower, a divergent-settling-convergent section, and the test section. The inlet and exit cross-sectional area of the divergent section was $25 \times 25 \mathrm{~cm}^{2}$ and $70 \times 70 \mathrm{~cm}^{2}$, respectively. The convergence ratio of the convergent section was 81.7. A honeycomb and a screen were installed inside the settling chamber to reduce the turbulence intensity of flow. Flow visualization was achieved by the integration of a laser-sheet (width $1 \mathrm{~mm}$ ) and the syringe-injected titanium tetrachloride $\left(\mathrm{TiCl}_{4}\right)$, which formed a mist of hydrogen chloride $(\mathrm{HCl})$ within the test section for light scattering.

The flow velocity was measured by a two-component forwardscattered laser-Doppler anemometry (LDA by TSI) [8,9]. An argonion laser (Coherent, $5 \mathrm{~W}$ ) served as the light source and a processor (TSI IFA-750) was utilized to process the Doppler signals. Seeding was accomplished by injecting aluminum oxide $\left(\mathrm{Al}_{2} \mathrm{O}_{3}\right)$ powder with an average diameter of $1 \mu \mathrm{m}$. Measurement of pressure was conducted by a static pressure tube connected to a transducer (VALIDYNE DP103-20) and an amplifier/display set (DP23). The static pressure tube was inserted into the flow from a slit at the top wall of test section, and was positioned both vertically and horizontally by a positioning platform. The analogue pressure signal was sampled and recorded through a PC-based data acquisition card (ADVANTEC PCL-818HG). The sampling rate for pressure measurement was $1 \mathrm{kHz}$.

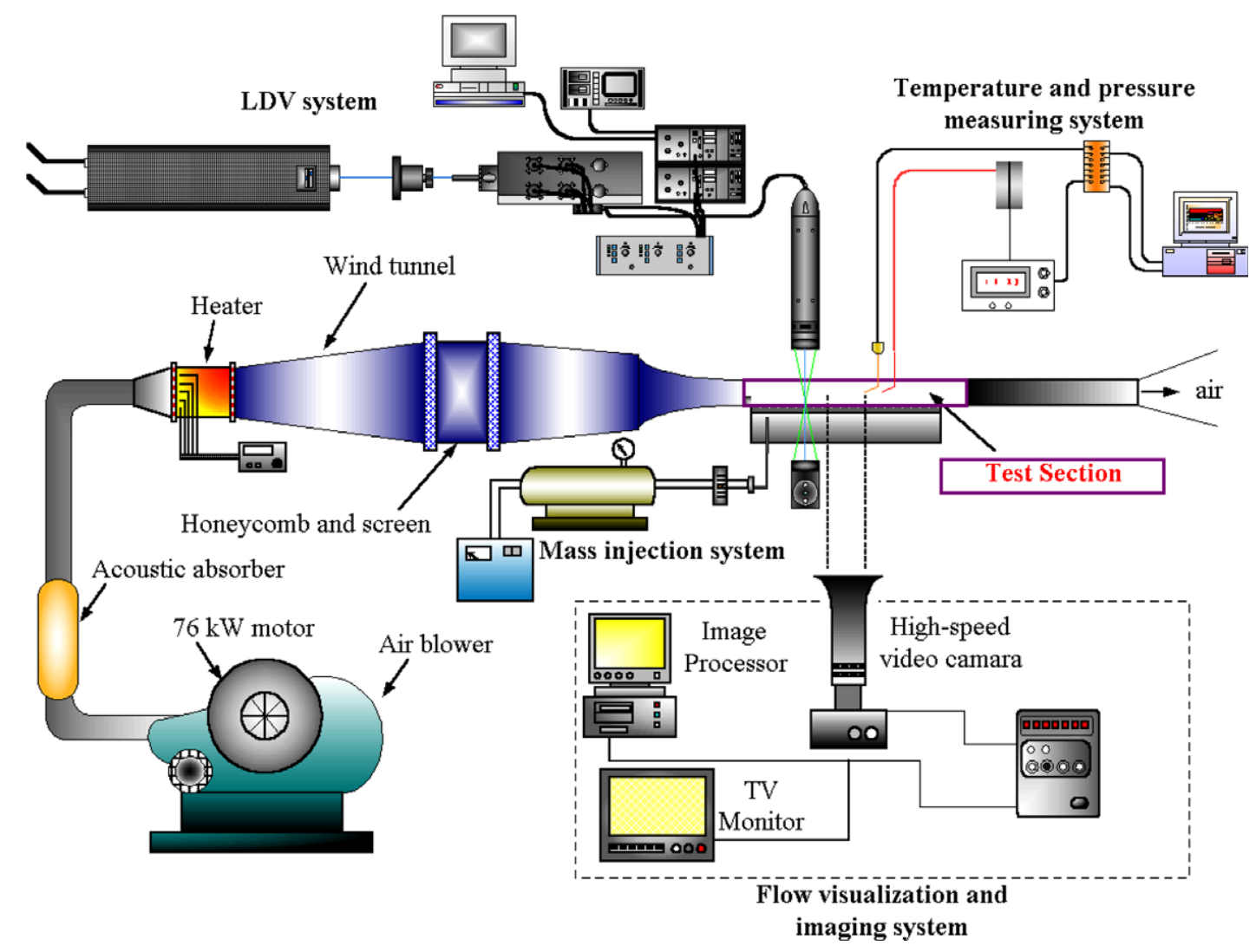

Fig. 2. Schematic diagram of the experiment setup. 


\subsubsection{Uncertainty analysis}

An analysis of uncertainty was conducted at two locations on the spanwise centerline plane $(z=0):(x, y)=(50 \mathrm{~mm}, 22 \mathrm{~mm})$ and $(250 \mathrm{~mm}, 22 \mathrm{~mm})$, for which $(x, y)=(0,0)$ was the root of backstep. Twenty sets of data, each comprising 1024 samples, were adopted for analysis. The data from LDA measurement was recorded over $180 \mathrm{~s}$ by adjusting the seeding concentration. This sampling duration was 100 times longer than the period of cyclic flow motion, which verified the statistically stationary nature of the results from LDA measurement. Due to the limitation of two-component LDA measurement and the asserted two-dimensionality of the flow at the spanwise centerline plane, the turbulent kinetic energy value $k$ was calculated by assuming that the spanwise component ( $z$-component) is zero. This assumption was verified by the symmetric spanwise distribution of turbulence properties about the $z=0$ plane. Based on 95\% confidence level, the maximum uncertainty of mean horizontal velocity, mean vertical velocity, turbulence intensity, the Reynolds stress and mean static pressure was $2.9 \%, 5.1 \%, 7.1 \%$, $9.9 \%$ and $3.4 \%$, respectively. The various bias uncertainties of LDA system in this work have been analyzed to be less than $1.5 \%$.

\subsection{Test conditions}

The wall injection velocity ratio $(M)$ is defined as:

$M=V_{\mathrm{w}} / U_{0}$

in which $U_{0}$ is the mean inlet velocity. The Reynolds number based on height of backstep $H\left(R e_{\mathrm{h}}\right)$ is defined as:

$R e_{\mathrm{h}}=U_{0} \mathrm{H} / \mathrm{v}$

in which $v$ is the kinematic viscosity. The mean superficial velocity of wall injection is defined as:

$V_{\mathrm{w}}=Q_{\mathrm{w}} / \Phi A_{\mathrm{p}}$

in which $Q_{\mathrm{w}}$ is the volume flow rate of wall injection, $\Phi$ is the porosity and $A_{\mathrm{p}}$ is the area of the porous base. The differential pressures across the porous base ranged from 5000 to $100,000 \mathrm{~Pa}$, which were over 1000 times of the maximum differential pressure measured within the flow. The uniformity of this superficial bleed velocity had been discussed by Yang and Kuo [9].

In this study, both the mean inlet velocity $\left(U_{0}\right)$ and the mean superficial velocity of wall injection $\left(V_{\mathrm{w}}\right)$ were selected to serve as the controlled parameters, and the corresponding wall injection velocity ratio $(M)$ was evaluated accordingly (Table 1 ). By conduct-
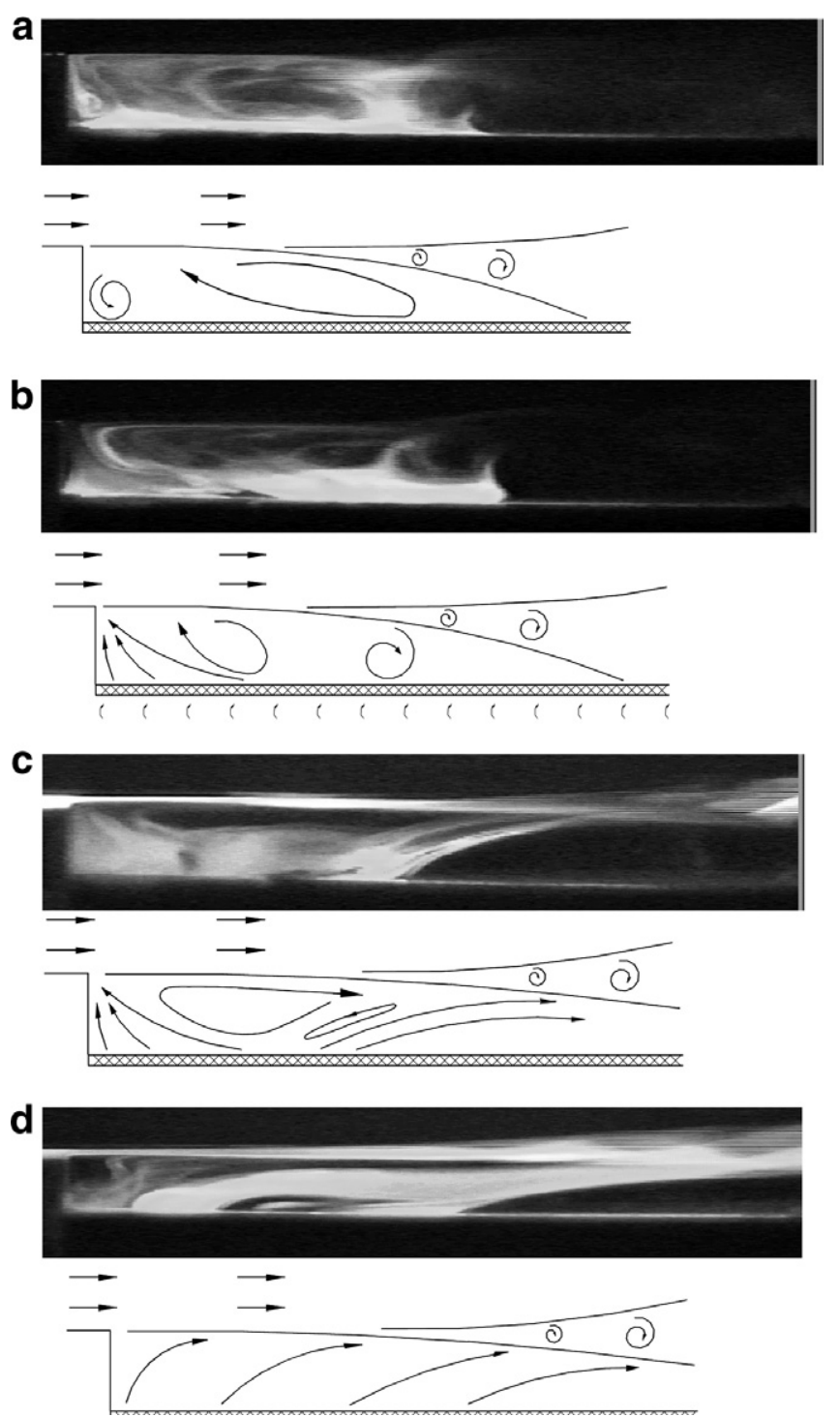

Fig. 3. Picture and schematic diagram of the flow in (a) pattern A: $U_{0}=2.9 \mathrm{~m} / \mathrm{s}$, $V_{\mathrm{w}}=0 \mathrm{~m} / \mathrm{s}, \quad M=0 \%$; (b) pattern B: $U_{0}=2.9 \mathrm{~m} / \mathrm{s}, \quad V_{\mathrm{w}}=0.068 \mathrm{~m} / \mathrm{s}, \quad M=2.34 \%$; (c) pattern C: $U_{0}=2.6 \mathrm{~m} / \mathrm{s}, \quad V_{\mathrm{w}}=0.116 \mathrm{~m} / \mathrm{s}, \quad M=4.46 \%$; and (d) pattern $\mathrm{D}$ : $U_{0}=2.1 \mathrm{~m} / \mathrm{s}, V_{\mathrm{w}}=0.17 \mathrm{~m} / \mathrm{s}, M=8.1 \%$.

Table 1

Test conditions for flow visualization: wall injection velocity ratio $M$ under various $U_{0}$ and $V_{\mathrm{w}}$

\begin{tabular}{|c|c|c|c|c|c|c|c|c|c|c|c|}
\hline \multirow[t]{2}{*}{$U_{0}(\mathrm{~m} / \mathrm{s})$} & \multirow[t]{2}{*}{$R e_{\mathrm{h}}$} & \multicolumn{10}{|c|}{ Mean superficial velocity of wall injection $V_{w}$} \\
\hline & & 0 & 0.034 & 0.048 & 0.068 & 0.082 & 0.102 & 0.116 & 0.136 & 0.150 & 0.170 \\
\hline & & $M$ & & & & & & & & & \\
\hline 2.1 & 2009 & 0.0000 & 0.0162 & 0.0229 & 0.0324 & 0.0390 & 0.0486 & 0.0552 & 0.0648 & 0.0714 & 0.0810 \\
\hline 2.6 & 2487 & 0.0000 & 0.0131 & 0.0185 & 0.0262 & 0.0315 & 0.0392 & 0.0446 & 0.0523 & 0.0577 & 0.0654 \\
\hline 2.9 & 2774 & 0.0000 & 0.0117 & 0.0166 & 0.0234 & 0.0283 & 0.0352 & 0.0400 & 0.0469 & 0.0517 & 0.0586 \\
\hline 3.2 & 3061 & 0.0000 & 0.0106 & 0.0150 & 0.0213 & 0.0256 & 0.0319 & 0.0363 & 0.0425 & 0.0469 & 0.0531 \\
\hline
\end{tabular}

Table 2

Pattern envelope under various test conditions

\begin{tabular}{|c|c|c|c|c|c|c|c|c|c|c|c|}
\hline \multirow[t]{2}{*}{$U_{0}(\mathrm{~m} / \mathrm{s})$} & \multirow[t]{2}{*}{$R e_{\mathrm{h}}$} & \multicolumn{10}{|c|}{ Wall injection velocity ratio } \\
\hline & & 0 & 0.034 & 0.048 & 0.068 & 0.082 & 0.102 & 0.116 & 0.136 & 0.150 & 0.170 \\
\hline 2.1 & 2009 & A & B & B & C & $\mathrm{C}$ & $\mathrm{C}$ & D & D & D & D \\
\hline 2.6 & 2487 & A & B & B & B & C & $C$ & $C$ & $C$ & D & $\mathrm{D}$ \\
\hline 2.9 & 2774 & A & A & B & B & B & B & C & $\mathrm{C}$ & D & $\mathrm{D}$ \\
\hline 3.2 & 3061 & A & A & A & B & B & B & B & C & D & $\mathrm{D}$ \\
\hline
\end{tabular}


ing flow visualization over a wide range of the parameters, a pattern envelope (Table 2 ) based on the wall injection velocity ratio $M$ was established. Four distinct flow patterns and their corresponding wall injection velocity ratios were identified accordingly. Based on the identified patterns, four specific sets of inlet and wall injection conditions, each corresponded to a respective pattern, were then selected for further quantitative measurements of the mean velocity field, pressure field and turbulence field. They were: (a) $U_{0}=2.9 \mathrm{~m} / \mathrm{s}, V_{\mathrm{w}}=0(M=0 \%)$ for pattern A (no wall injection), (b) $U_{0}=2.9 \mathrm{~m} / \mathrm{s}, V_{\mathrm{w}}=0.068 \mathrm{~m} / \mathrm{s} \quad(M=2.34 \%)$ for pattern $\mathrm{B}$, (c) $U_{0}=2.6 \mathrm{~m} / \mathrm{s}, V_{\mathrm{w}}=0.116 \mathrm{~m} / \mathrm{s}(M=4.46 \%)$ for pattern $\mathrm{C}$, and (d) $U_{0}=2.1 \mathrm{~m} / \mathrm{s}, V_{\mathrm{w}}=0.17 \mathrm{~m} / \mathrm{s}(M=8.1 \%)$ for pattern $\mathrm{D}$.

Note the ratio of cross-sectional area of the inlet channel $\left(A_{0}\right)$ to that of the porous base $\left(A_{\mathrm{p}}\right)$ was 0.1 , which implied that the mass flow rate ratio of wall injection to inlet flow was 10 times of the wall injection velocity ratio. Identical tendency of the flow behavior was concluded by considering either the inlet/base area ratio or the wall injection velocity ratio.

Among all the inlet conditions tested, the shape factors vary in the range 1.31-1.37. According to Pitz and Dailey [14], the shape factor for turbulent flow is about 1.3. The inlet flow in this study was in the transitional regime toward turbulence. In all cases, the shape factors of flow upstream of the backstep were almost identical and the inlet flow was isotropic. The flow downstream of the backstep was transitional, though, and the turbulent characteristic became more prominent as the flow went further downstream. It was also observed that the turbulent characteristic was suppressed as the wall injection was initiated.

One of the most distinctive characteristics of the separationreattachment flow over a backstep is the reattachment length. In this study, the point of reattachment is defined as the streamwise location at which the horizontal velocity is zero. To locate this point, the horizontal velocity was measured $1 \mathrm{~mm}$ above the base downstream of the backstep. For patterns $A$ and $B$, the reattachment lengths $\left(X_{\mathrm{r}}\right)$ were measured to be $4.47 \mathrm{H}$, whereas the reattachment points were not accessible in patterns $C$ and $D$. The value $4.47 \mathrm{H}$ was selected as the common reattachment length for the four flow patterns in the following analysis.

\section{Results and discussion}

\subsection{Flow visualization}

Two streams with opposite flow directions were observed within the recirculation region. One stream, diverted from the shear layer after reattachment, flowed toward the backstep along the base wall and formed the clockwise-rotating recirculation zone. It swept the fluid near wall upstream. The strength of upstreamsweeping momentum was produced by the low pressure at the recirculation zone, and the strength was found to increase by increasing the Reynolds number of the inlet flow. Initiation of wall injection was found to decrease the upstream-sweeping momentum of flow within the recirculation zone due to the effects of entrainment and diffusion. Fluid injected from the base into the recirculation zone was swept upstream and accumulated at the corner of backstep. By increasing the flow rate of wall injection, pressure level in the recirculation zone was increased. The strength of upstream-sweeping momentum was decreased accordingly, which produced various downstream-flowing tendencies and distinct flow patterns.
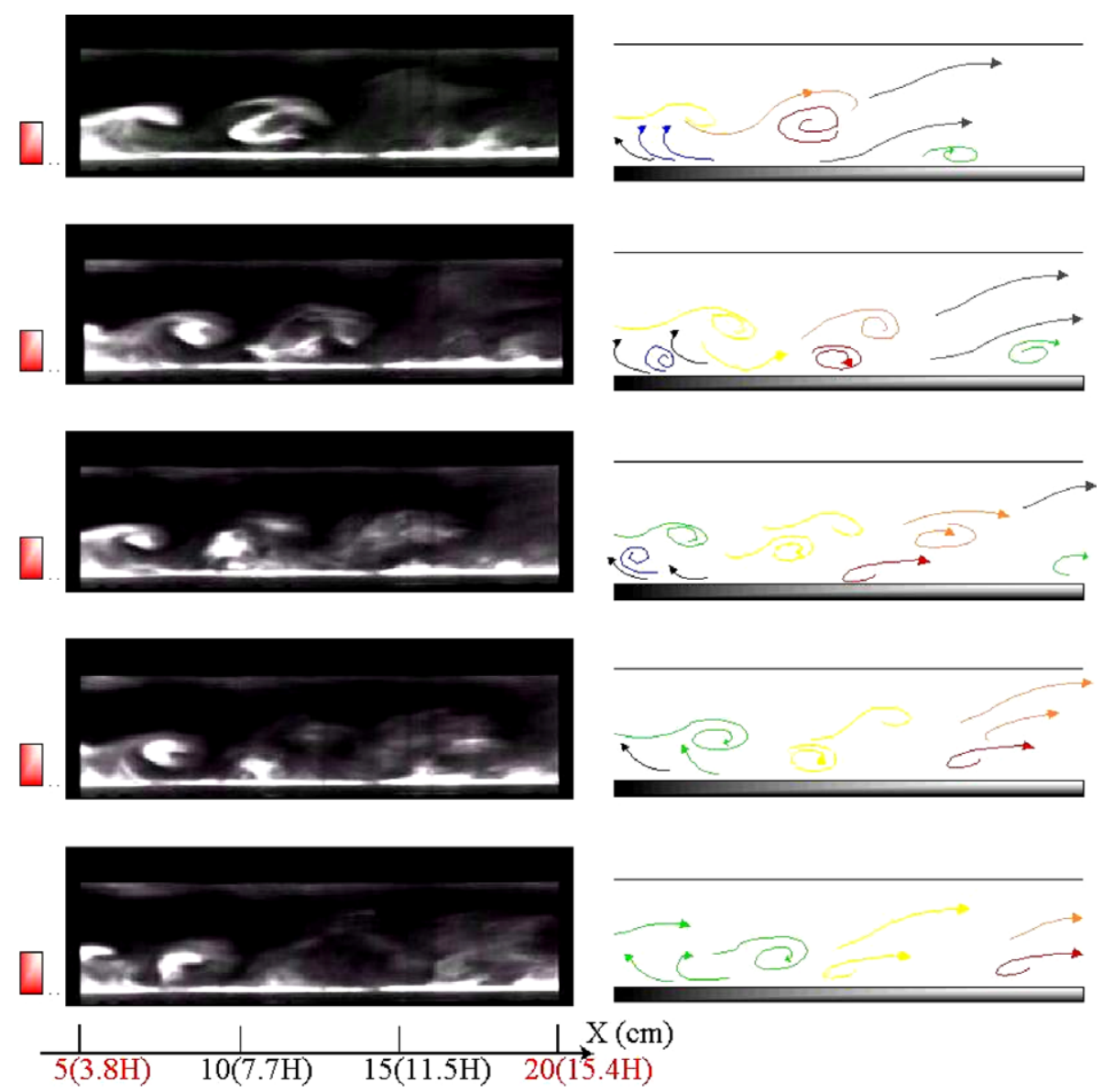

Fig. 4. Pictures and schematic diagrams of flow in pattern A at $5-20 \mathrm{~cm}$ downstream of the backstep (times step $=0.1666 \mathrm{~s}$ ). 
Considering a large $U_{0}$ and a relatively minor wall injection, with a limiting proportion of zero, a significant recirculation vortex was identified. Following the shear layer the fluid touched the base and rolled upstream. The upstream-moving flow was blocked by the backstep and separated again, by which a corner eddy was produced. The flow pattern was designated pattern $\mathrm{A}$, as demonstrated in Fig. 3(a).

By increasing the wall injection velocity ratio, the injected fluid was swept toward the backstep and accumulated at the corner. Under the condition that the downstream-flowing momentum produced by injection was not significant enough to balance the recirculation (pattern $\mathrm{B}$ ), fluid accumulated at the corner was pressed toward the shear layer near the tip of backstep (Fig. 3(b)). A triangular zone was identified near the corner, and the injected mass was observed to join the shear layer through an exit at top of the triangle.

Further increasing the wall injection velocity ratio transformed the flow pattern into pattern C (Fig. 3(c)). The exit at the triangular zone was no longer wide enough to drain the extra mass accumulated at the corner. The downstream-flowing momentum was able to overcome the recirculation, and part of the fluid injected began to flow directly downstream through the reattachment region. The entire flow structure downstream of the backstep was elevated. Although the recirculation zone was occasionally recognizable, it was observed to shrink into a flatter form. The downstream-flowing momentum became dominant by increasing the mass injection velocity ratio above certain values. The injected fluid flew directly downstream and the flow structure under this pattern (Fig. 3(d), pattern D) was relatively simple. The multiple characteristics of the original separation-reattachment flow were no longer recognizable.

Totally 40 sets of inlet and wall injection conditions were tested and observed to establish the pattern envelope in Table 2 . The four patterns emerged in turn by increasing the wall injection ratio. Contrary to the relatively large $U_{0}$ with minor $V_{\mathrm{w}}$ for pattern A, a relatively small $U_{0}$ with large $V_{\mathrm{w}}$ transformed the flow structure into pattern $\mathrm{D}$. The influence of the Reynolds number was also revealed in Table 2 . Since the Reynolds number implies the ratio of flow inertia to viscous effect, the higher the Reynolds number, the more dominant the inertia of the inlet stream. The relatively minor viscous effect under higher Reynolds number indicated weaker interactions between the inlet flow and wall injection, which made pattern A more favorable since it shared similar characteristics with the typical separation-reattachment flow. The flow behavior of other similar studies was able to be categorized and predicted according to the established envelope. For instance, the work by Yang and Kuo [9] corresponded to a wall injection velocity ratio of 0.005 , which fell into the regime of pattern $A$, and the predicted pattern coincided with the streamline contour in [9].

Detailed flow structure around the reattachment region and the corner of backstep under the four flow patterns were further investigated. Inspections of the transient flow structure were first conducted between the locations $x=50 \mathrm{~mm}(3.8 H)$ and $x=200 \mathrm{~mm}$ $(15.4 H)$ downstream of the backstep, which was around the reattachment region (Fig. 4). The reattachment region under pattern A was found to locate about five times the step height $(5 \mathrm{H})$
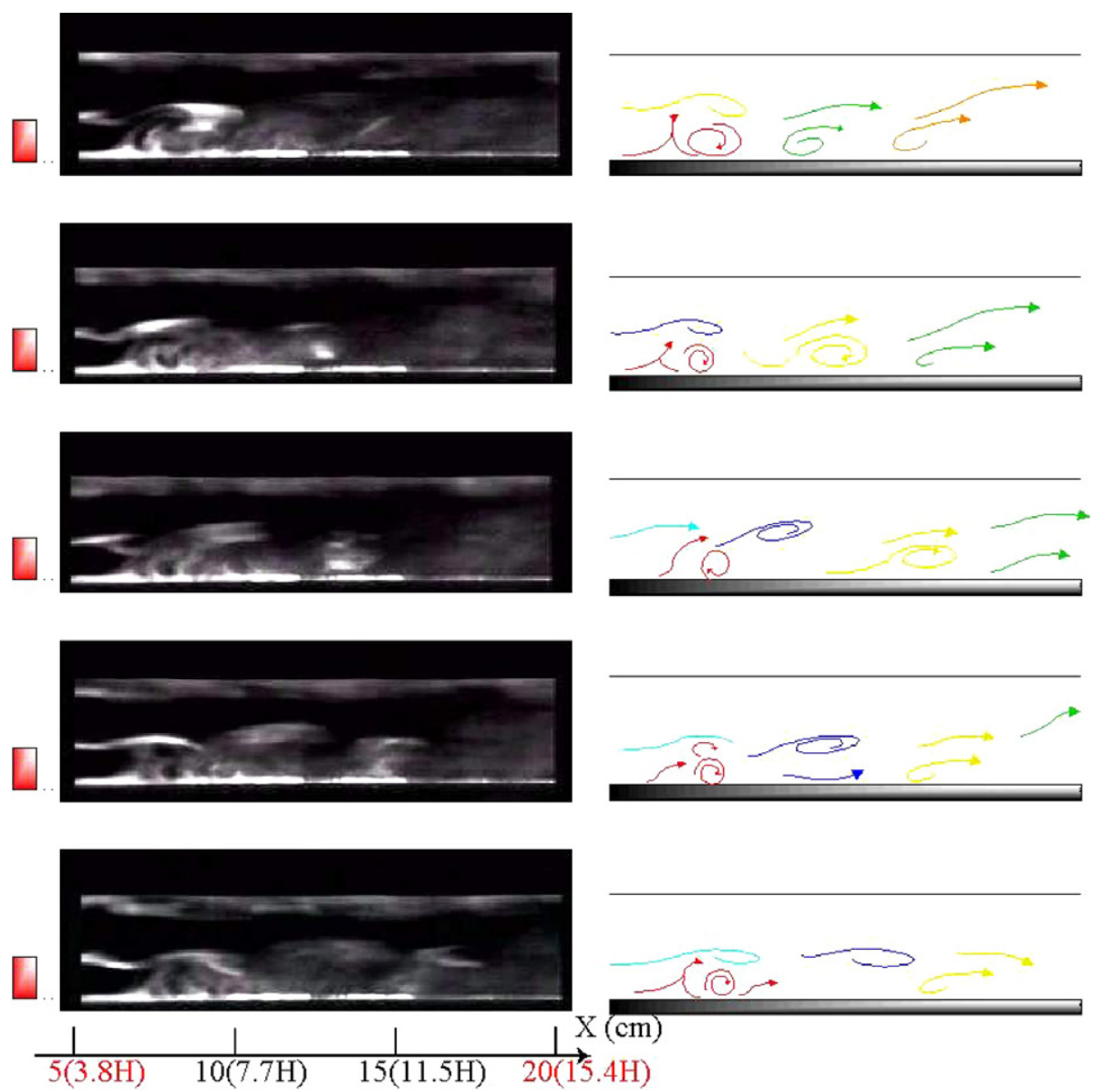

Fig. 5. Pictures and schematic diagrams of flow in pattern $B$ at $5-20 \mathrm{~cm}$ downstream of the backstep (times step $=0.1666 \mathrm{~s}$ ). 
downstream of the backstep. The shedding vortices were observed to impact the base in a clockwise-rotating manner and roll into the recirculation bubble.

The reattachment region located approximately $8 H$ downstream of the backstep under pattern B (Fig. 5). An almost stagnant vortex was developed adjacent to the reattachment region, which indicated that the upstream-sweeping momentum of recirculation was neutralized to certain extent due to the initiation of wall injection. However, the mass injected was not significant enough to drain directly downstream through the reattachment region. Further increasing the wall injection velocity ratio transformed the flow structure into pattern $C$ and the reattachment region was observed to locate about $12 \mathrm{H}$ downstream of the backstep (Fig. 6). The reattachment region was occasionally unrecognizable since the increased wall injection was capable of leaking directly downstream. Note that instead of rolling upstream into the recirculation bubble, the vortex adjacent to the reattachment region was forced by the mass injected to roll downstream. The reattachment region became completely unrecognizable under pattern $\mathrm{D}$ (Fig. 7). The shedding vortices were not able to approach the base since the entire flow structure was elevated by the injected mass.

The flow visualization conducted near the corner of backstep also revealed distinct features for each pattern. A corner eddy was developed since the recirculation flow was not capable of turning perpendicularly along the corner (Fig. 8(a)). The eddy was then suppressed downward by the accumulated fluid. After a certain period, adequate momentum of the accumulated fluid was established, the eddy was then destructed, and the fluid was able to escape the corner through the tip of backstep. The process repeated periodically at approximately $2.5 \mathrm{~Hz}$. Increasing of wall injection in pattern $B$ extended the recirculation zone upstream and compressed the corner eddy (Fig. 8(b)). The momentum carried by recirculation into the corner eddy was decreased due its interaction with wall injection. Consequently, the frequency at which the fluid escaped through the tip of backstep was lower than that in pattern $A$.

The corner eddy no longer existed in patterns $C$ and D since the injected mass was capable of filling the entire volume adjacent to the backstep. A stretched upside down U-shaped flow structure (Fig. 8(c)) was observed between the escape flow immediately adjacent to the backstep and the recirculation bubble, which implied a further transition into pattern D. In pattern $D$, it was observed that most injected fluid flew directly downstream along the base (Fig. $8(\mathrm{~d})$ ). The stretched U-shaped flow in pattern $\mathrm{C}$ was elevated and formed a stagnant region as the dark zone in Fig. 8(d).

\subsection{Pressure field}

The distributions of mean pressure coefficient along the spanwise centerline plane $(z=0)$ for each flow pattern were demonstrated in Fig. 9. The mean pressure coefficient $C_{p}$ was defined as: $C_{p}=\frac{P-P_{0}}{\frac{1}{2} \rho U_{0}^{2}}$

Note that the reference pressure $P_{0}$ was measured $30 \mathrm{~mm}$ upstream of the backstep and $29 \mathrm{~mm}$ above the bottom wall surface of inlet channel.

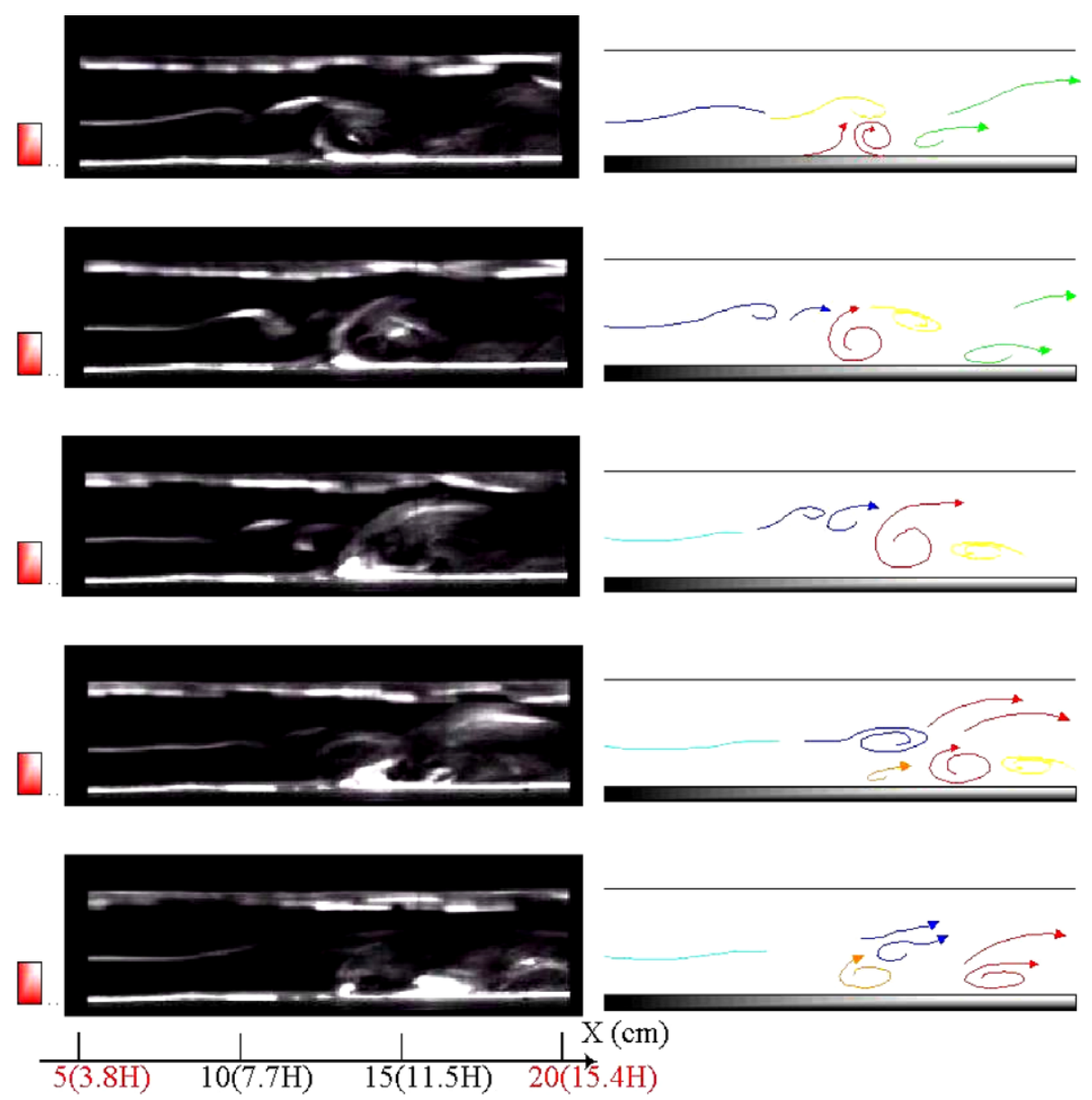

Fig. 6. Pictures and schematic diagrams of flow in pattern $C$ at $5-20 \mathrm{~cm}$ downstream of the backstep (times step $=0.1666 \mathrm{~s}$ ). 
The pressure within the recirculation zone in pattern A was significantly smaller than elsewhere in the flow (Fig. 9(a)). A locally minimum pressure was measured at the corner of backstep. For the typical separation-reattachment flow over a backstep, an adverse pressure gradient was established by the low-pressure zone within the recirculation bubble. The adverse gradient caused the flow to separate at the tip of backstep at which the inertia of flow downstream was relatively small. The pressure was recovered gradually downstream as shown in the figure. Contrary to the pressure distribution in pattern A, a higher pressure level was established near the corner of backstep due to accumulation of the injected mass. Note also the pressure level within the recirculation zone in pattern $B$ was generally higher than that in pattern A, which produced weaker adverse pressure gradient and decreased strength of recirculation.

An even higher pressure level was established within the recirculation zone in pattern C (Fig. 9(c)). The adverse pressure gradient was almost diminished, which was consistent with the result from flow visualization that the mass injected was capable of leaking directly downstream. For pattern D (Fig. 9(d)), the streamwise pressure gradient became even more favorable for fluid to flow directly downstream, which again verified the pattern in Fig. 8(d). The region with maximum pressure in Fig. 9(d) was also found to correspond to the stagnant region in Fig. 8(d). The smooth distribution of $C_{p}$ along the wall-normal direction in Fig. 9 implied that the flow was essentially two-dimensional. The fact again verified the accountability of two-dimensional LDA measurement conducted at the spanwise centerline $(z=0)$ plane at which no significant wall effect was observed.

\subsection{Mean velocity and turbulence field}

Due to the intense mixing of momentum within the recirculation bubble and along the shear layer, distinct distributions of velocity components were revealed for the four flow patterns. In this part of the analysis, the designated common reattachment length $X_{\mathrm{r}}=4.47 \mathrm{H}$ was adopted to normalize the streamwise location downstream of the backstep for the four flow patterns. The mean horizontal velocity field ( $x$-direction) of each flow pattern was shown in Fig. 10, in which the effect of base injection was explicitly demonstrated. Since the LDA measurement was conducted over a sufficiently long time interval, the profile of statistically averaged horizontal velocity was essentially similar to that of the horizontal momentum. For patterns $\mathrm{A}$ and $\mathrm{B}$, a reverse velocity was observed around the location $(x, y)=\left(0.45 X_{\mathrm{r}}, 0.5 \mathrm{H}\right)$, which indicated an intact recirculation structure. For patterns $C$ and $D$, the reverse velocity field was less significant, which coincided with the distribution of pressure field. A large velocity gradient along $y$-direction was observed near the tip of backstep, which produced increased turbulent kinetic energy. The velocity distribution was gradually recovered downstream and behaved as that of a general channel flow. Note that the value of $U / U_{0}$ was found to be larger than 1.0 at the outer edge of boundary layer. The phenomenon was attributed to the compression of flow path formed between the boundary layer and the top wall of test section. The more mass injected from the base, the narrower the path became, and the flow velocity through the path had to be increased to comply with the conservation of mass.
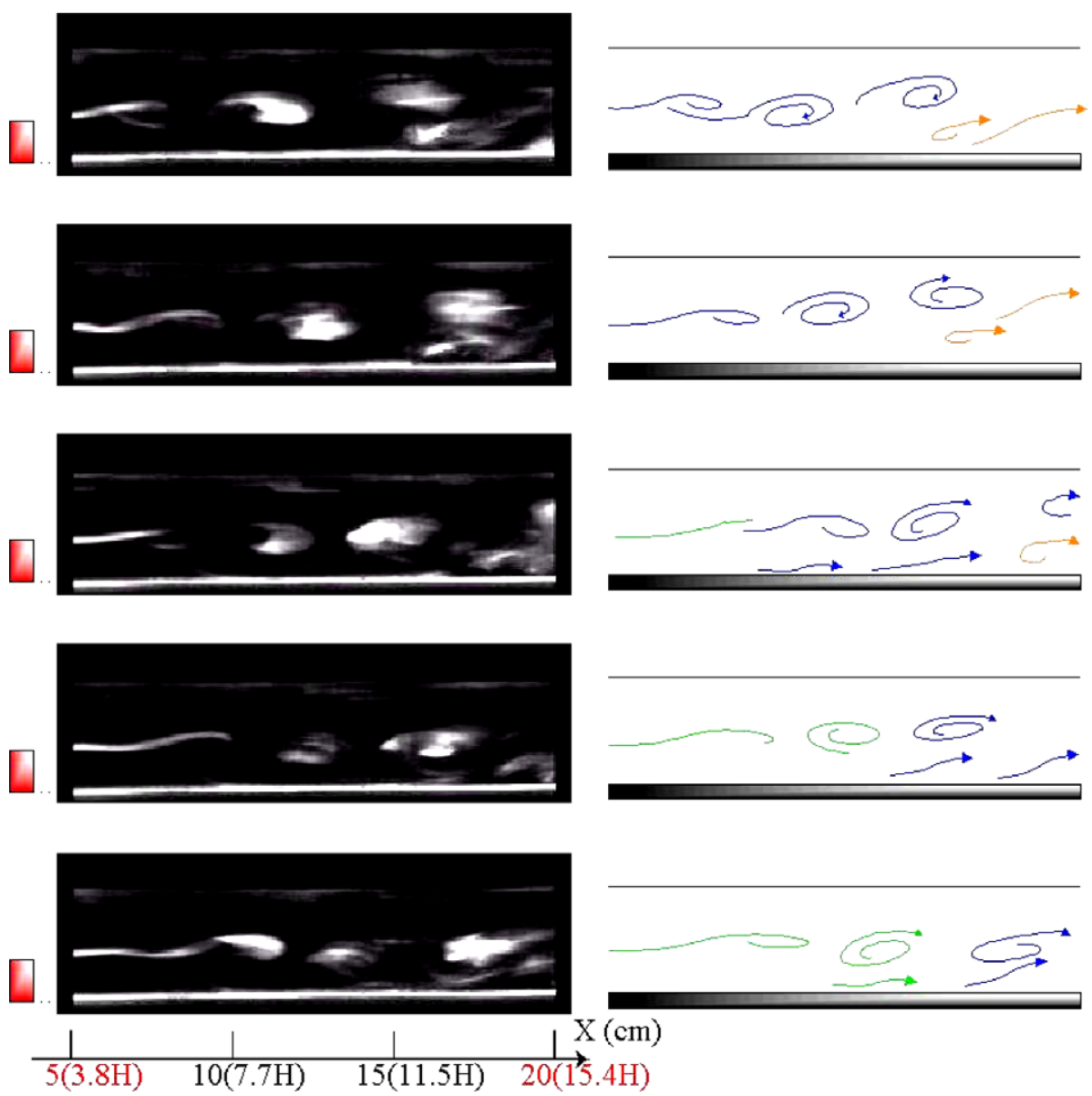

Fig. 7. Pictures and schematic diagrams of flow in pattern $D$ at $5-20 \mathrm{~cm}$ downstream of the backstep (times step $=0.1666 \mathrm{~s})$. 

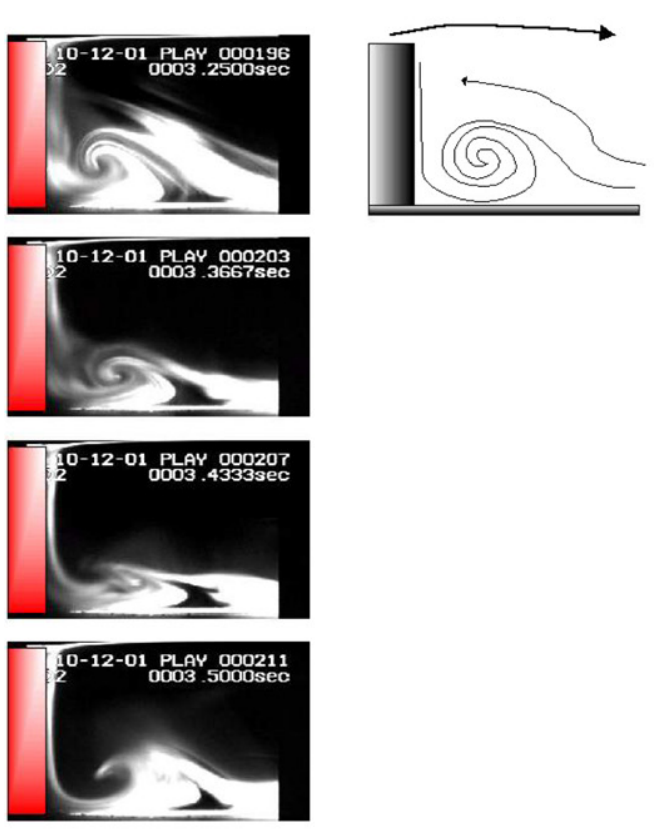

(a) pattern $\mathrm{A}$
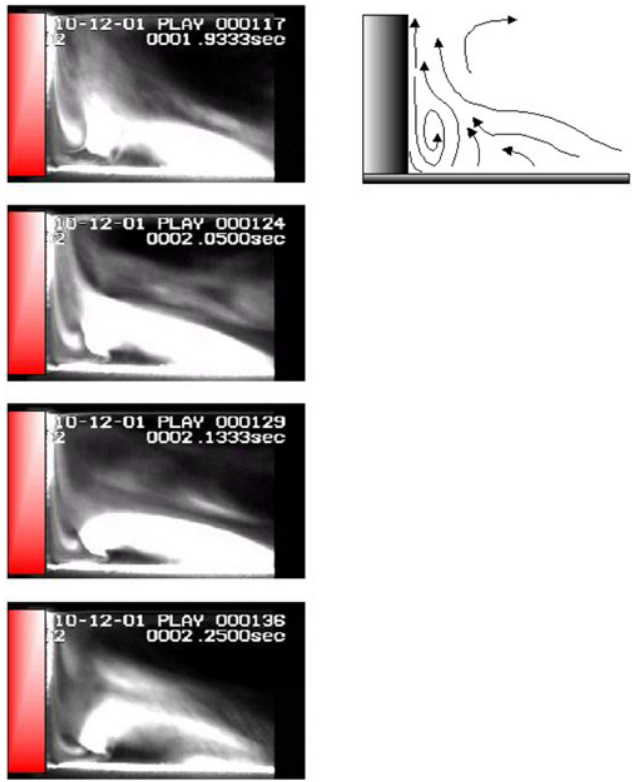

(c) pattern $\mathrm{C}$
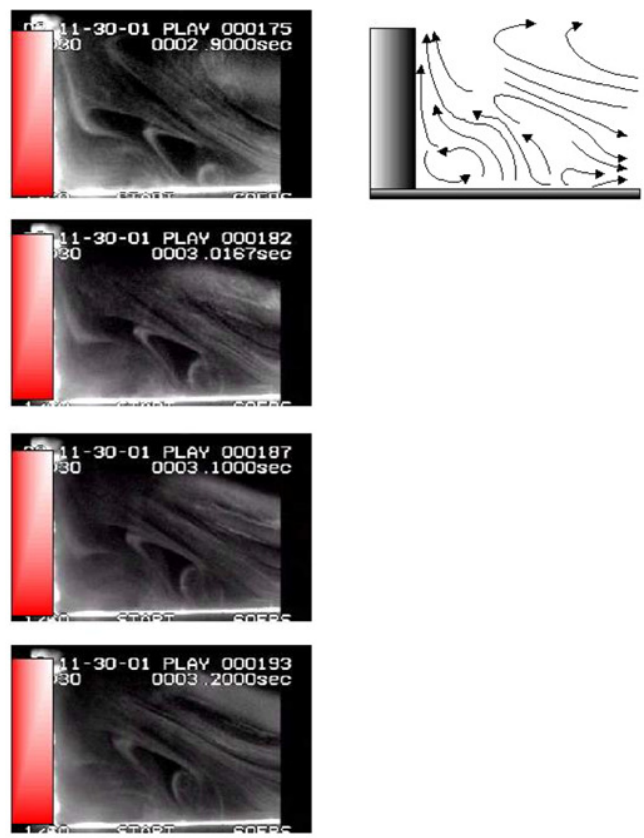

(b) pattern B
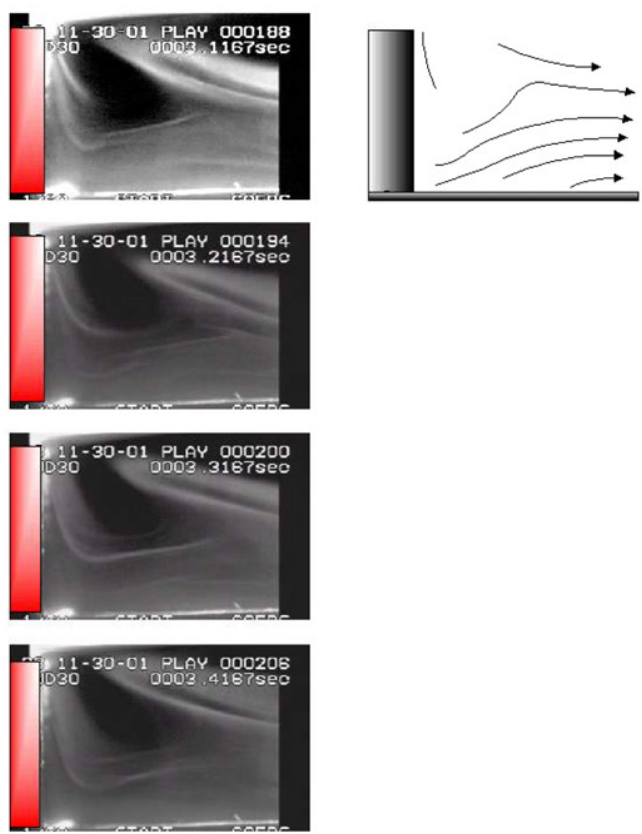

(d) pattern $\mathrm{D}$

Fig. 8. Pictures and schematic diagrams of flow near the corner of backstep for patterns from A to D.

The distributions of mean vertical velocity ( $y$-direction) for the four flow patterns were shown in Fig. 11. The mean vertical velocity was observed to be uniformly upward at $x=0.15 X_{\mathrm{r}}$ due to the initiation of base injection for patterns B, C and D. At regions further downstream of the backstep, such as the redeveloped boundary layer, distinct distributions of vertical velocity were also observed between pattern A and the other three patterns with base injection. A region with upward velocity was observed in the upstream half of the recirculation bubble for patterns $A$ and $B$. However, the vertical velocity field for patterns A and B were mostly downward, which was identical to that of the typical separation-reattachment flow over a backstep. On the other hand, due to the increased base injection in patterns $C$ and $D$, the vertical velocity field was mostly dominated by upward direction. The vertical component of velocity was decreased as the flow moved gradually downstream. Note that no significant distinction was observed between the profiles of vertical velocity for patterns $C$ and $D$. The similarity was attributed to the saturated mixing between the recirculated and injected fluids, and was consistent with the trends revealed in Fig. 3.

Since the Reynolds number of the flow studied in this work fell in the regime of transition toward turbulence, both laminar and turbulence characteristics were revealed simultaneously. The 

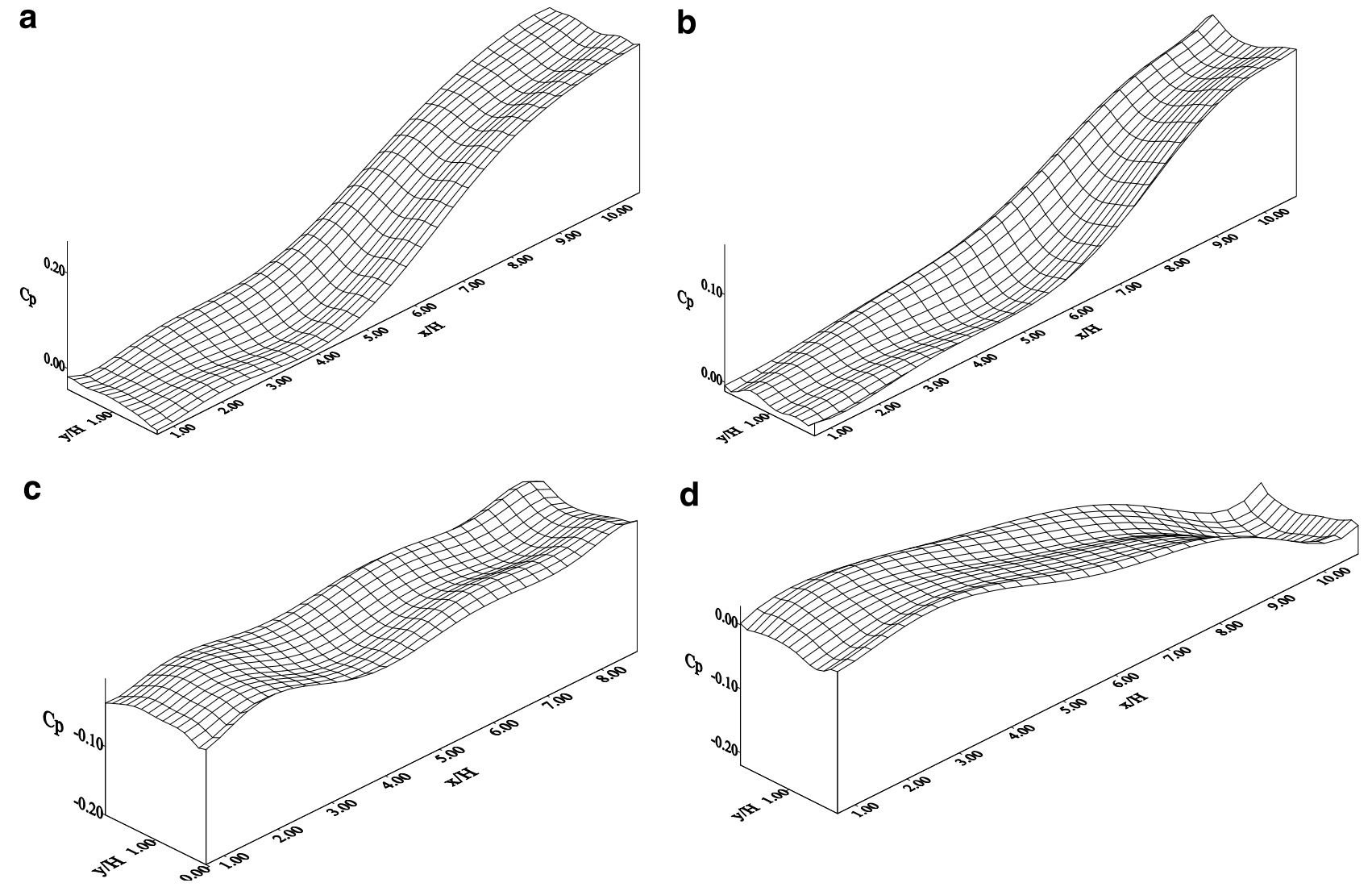

Fig. 9. Pressure distribution for patterns from A to D.

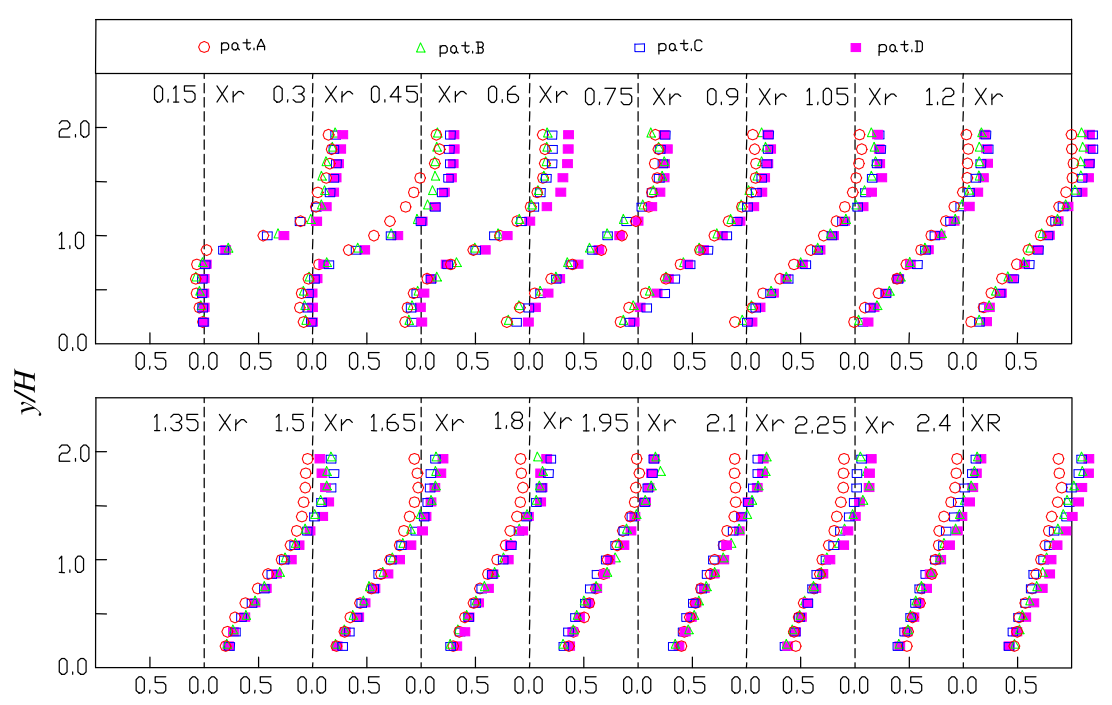

Fig. 10. Distribution of mean horizontal velocity $U / U_{0}$.

behavior of flow was found to alternate among several quasi-steady non-turbulent states. Consequently, both the shear layer and recirculation bubble were non-turbulent and non-isotropic, which was verified by the fact that the $u_{\mathrm{rms}} \fallingdotseq 0.5 U_{0}$ and $v_{\mathrm{rms}}$ was negligible at these regions.

The normalized Reynolds stress for each flow pattern was shown in Fig. 12. For pattern A, a large value of the Reynolds stress was measured at $(x, y)=\left(0.15 X_{\mathrm{r}}, 1 H\right)$, which was near the tip of backstep. The significant Reynolds stress was attributed to the mixing process between the entrained fluid from recirculation zone and the shear layer. The wall-normal ( $y$-direction) location with locally maximum Reynolds stress at each streamwise measuring stage was found to move toward the base gradually as the flow moved downstream. The distribution was consistent with the configuration of shear layer as it bent toward the base. For patterns $\mathrm{B}, \mathrm{C}$ and $\mathrm{D}$, the magnitudes of the Reynolds stress at $(x, y)=\left(0.15 X_{\mathrm{r}}, 1 H\right)$ were less than that in pattern $\mathrm{A}$. Instead of bending directly toward the base, the shear layer in patterns $B, C$ and $\mathrm{D}$ was lifted by the mass injected. The distinction implied that the intensity of mixing was decreased, and the inlet stream was 


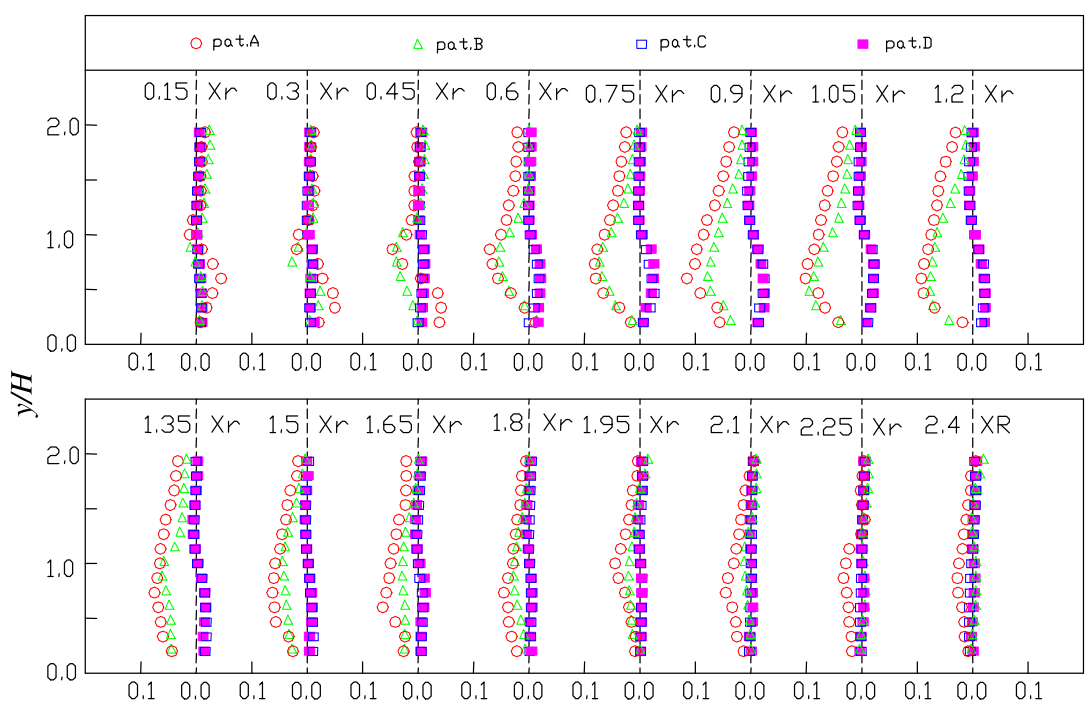

Fig. 11. Distribution of mean vertical velocity $V / U_{0}$.

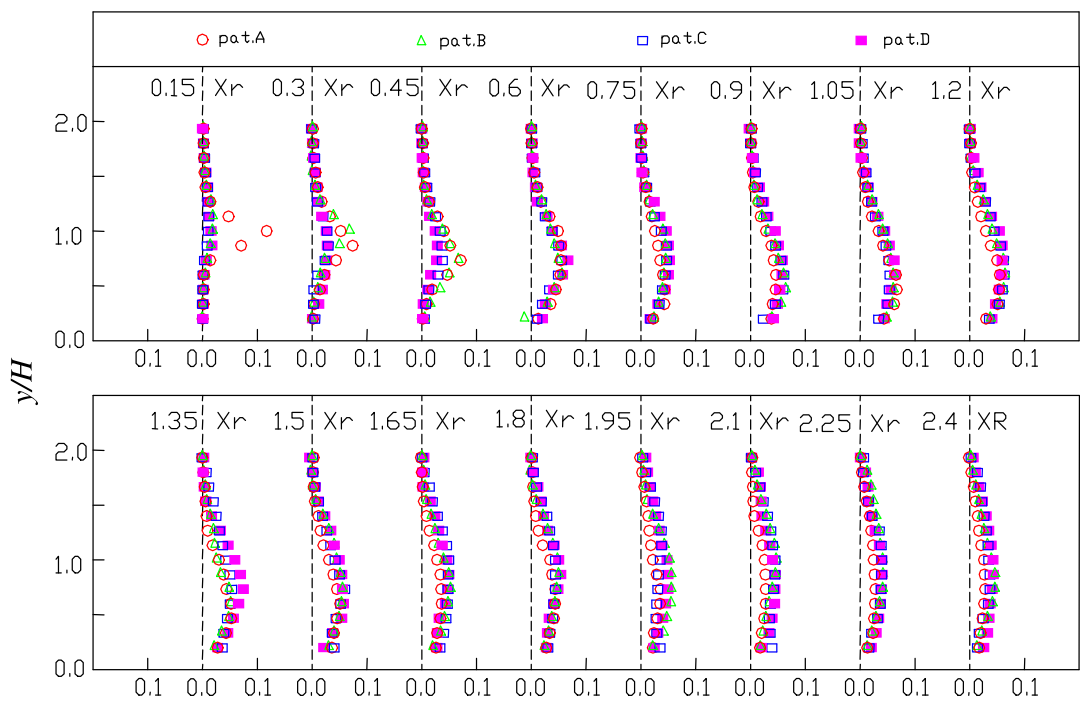

Fig. 12. Distribution of Reynolds stress $-3 \overline{u v} / U_{0}^{2}$.

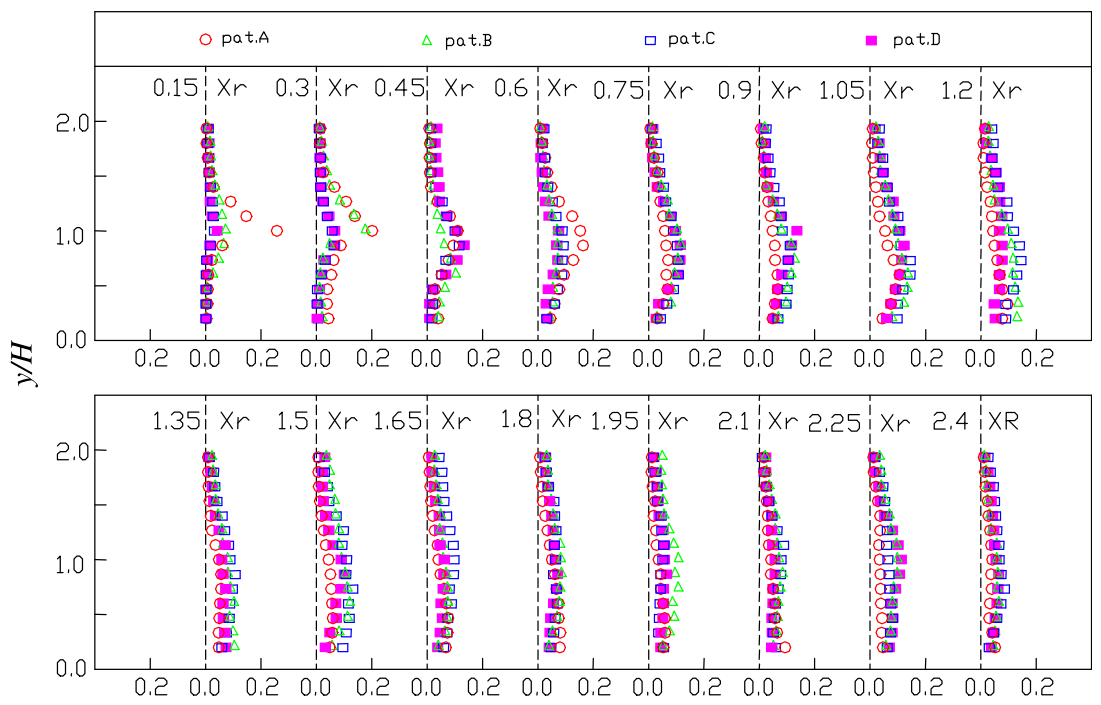

Fig. 13. Distribution of turbulent kinetic energy $k / U_{0}^{2}$. 
forced to accelerate and mix with the injected fluid further downstream. Note that contrary to the significant turbulence characteristic possessed by the shear layer, the fluid injected was screened by the porous plate and its velocity was almost negligible with respect to the inlet stream. The Reynolds number of the flow injected was relatively small, and so was its turbulent kinetic energy.

Based on the visualization of flow structure, the injected fluid was found to accumulate at the corner of backstep and flow into the shear layer via an exit near the tip of backstep. For patterns B, C and D, the level of Reynolds stress within the shear layer near the tip was decreased by the base injection which carried relatively minor turbulent kinetic energy. The decreasing effect was limited within the region near the streamwise stage $x=0.15 X_{\mathrm{r}}$ under pattern $\mathrm{B}$, in which the amount of injected fluid was small. However, the more the base injection, the wider the exit via which the injected fluid leaked, and the region affected by the decreasing effect extended toward the streamwise stage $x=0.45 X_{\mathrm{r}}$ in patterns $C$ and $\mathrm{D}$.

Identical trends were observed in the distributions of both the Reynolds stress and the turbulent kinetic energy (Fig. 13) for the four flow patterns. The transport of turbulent kinetic energy was also explicitly shown in Fig. 13. Generally, the turbulent kinetic energy was concentrated around the wall-normal location $y=1 \mathrm{H}$ at the streamwise stage $x=0.15 X_{\mathrm{r}}$. The mechanism of diffusive transport made the concentrated turbulent kinetic energy spread vertically as the flow moved downstream. The diffused turbulent kinetic energy was then gradually dissipated due to the existence of viscosity. At regions further downstream, such as the streamwise stage $x=2.4 X_{\mathrm{I}}$, the distribution of turbulent kinetic energy became more vertically uniform by diffusion, and the magnitude was decreased by dissipation.

The normalized turbulent normal stresses $\overline{u u}, \overline{v v}$ and shear stress $\overline{u v}$ with respect to the turbulent kinetic energy $k$ were summarized in Fig. 14 for analyzing the turbulence structure. These parameters, along with the mean horizontal velocity $U$, were plotted at two streamwise stages: $x=0.15 X_{\mathrm{r}}$ (recirculation zone) and $x=2.1 X_{\mathrm{r}}$ (further downstream). The anisotropic nature of the flow studied was demonstrated by the distinction revealed between the magnitudes of $\overline{u u} / k$ and $\overline{v v} / k$. In pattern A (Fig. 14(a)), the

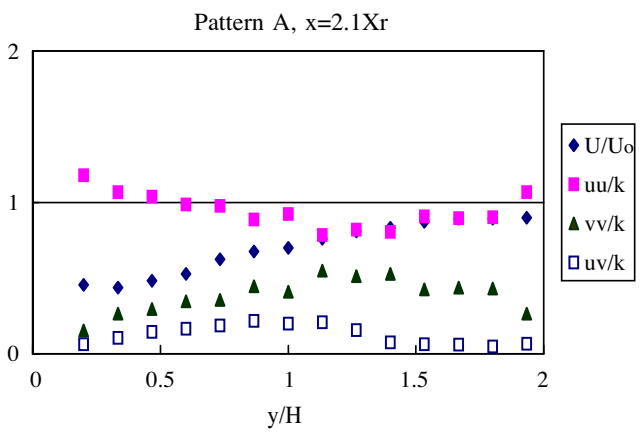

b
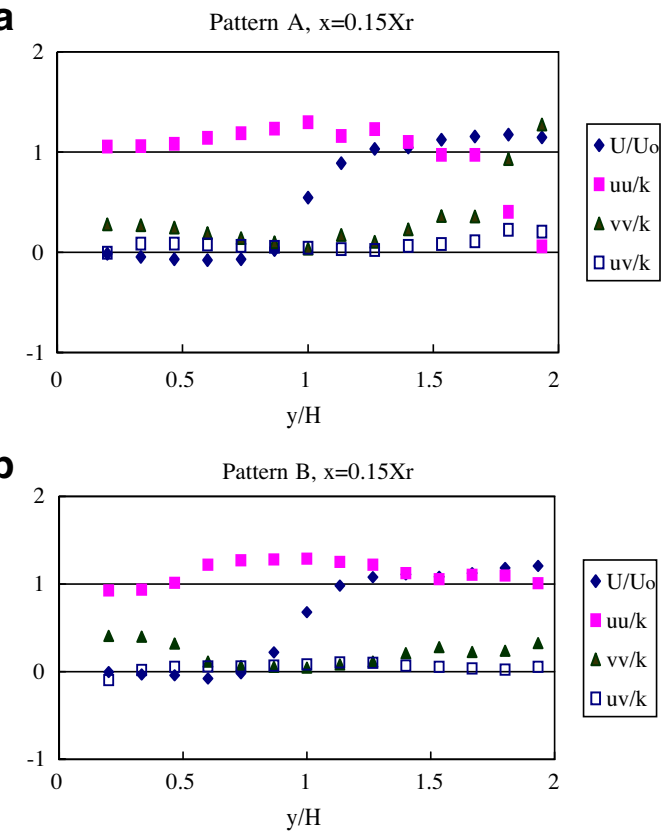

C
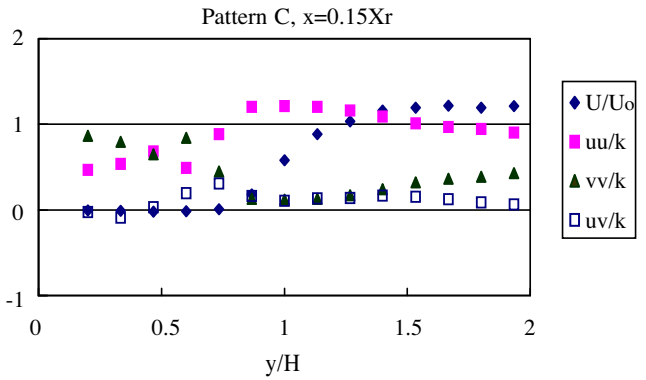

d

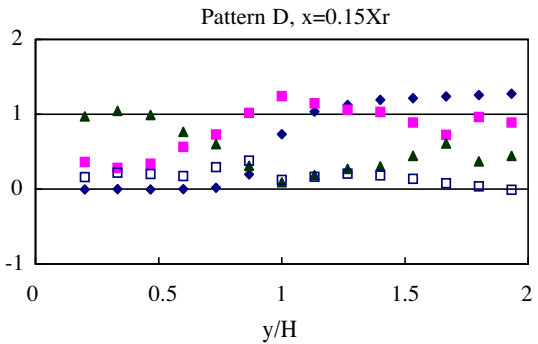

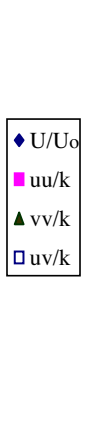
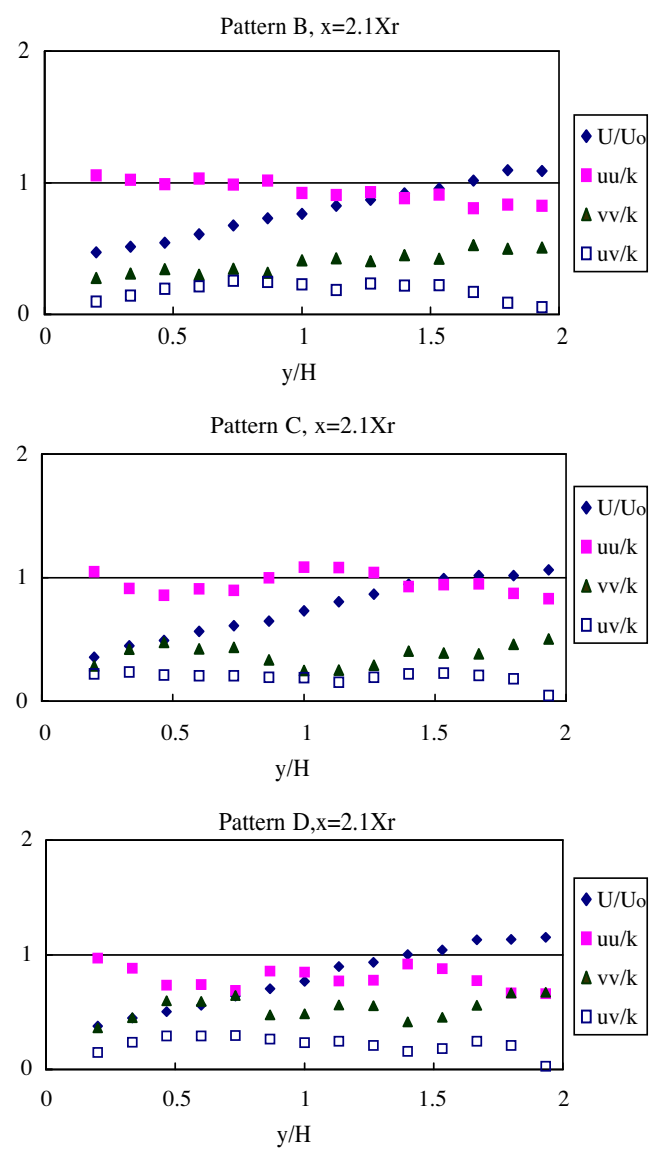

Fig. 14. Turbulence structure for patterns from $A$ to $D$. 
magnitude of $\overline{u u} / k$ was generally larger than that of $\overline{v v} / k$, especially near the wall-normal location $y=1 H$, which implied the major contribution of horizontal fluctuation to the turbulent kinetic energy. The fact was consistent with the nature of free mixing layer in which the turbulent kinetic energy was mostly contributed by the horizontal velocity gradient. On the other hand, the wall-normal location with maximum $\overline{u u} / k$ was shifted toward the bottom wall at the region downstream. The turbulent kinetic energy was mainly produced by the horizontal velocity gradient at the boundary layer adjacent to the bottom wall.

The weighting of vertical fluctuation was increased within the recirculation zone near the corner of backstep by increasing the wall injection velocity ratio (patterns $\mathrm{B}, \mathrm{C}$ and $\mathrm{D}$ ). Intense mixing along the wall-normal direction was produced by the fluid injected vertically from the base. The weighting of vertical fluctuation gradually achieved the level of its horizontal counterpart at the region downstream, which made the flow approach the status of isotropic turbulence.

\section{Conclusions}

The behavior of a separation-reattachment flow over a backstep with base injection downstream in the transitional flow regime of $R e_{\mathrm{h}}=2009-3061$ was analyzed both qualitatively and quantitatively. Four distinct flow patterns (A, B, C and D) were categorized and the wall injection velocity ratio was identified as a dominant criterion. By increasing the wall injection velocity ratio, the flow pattern was shifted sequentially from $A$ to $D$, and distinct flow behavior was observed within the recirculation vortex, at the reattachment region, and adjacent to the corner of backstep.

Based on the evaluation of mean pressure coefficient, the connection between flow pattern and pressure field was more explicitly demonstrated. A low-pressure zone was maintained within the recirculation bubble in pattern $A$ since the base injection was relatively minor. By gradually increasing the wall injection velocity ratio, the pressure near the corner of backstep was increased due to the accumulation of injected fluid. The inherent adverse pressure gradient was decreased and the strength of recirculation was weaker. Due to the increased base injection which destructed the recirculation mechanism, the streamwise pressure gradient in pattern D became more favorable for fluid to flow directly downstream.

Through the measurement of mean velocity field, the existence of an intact recirculation vortex was verified for patterns A and B.
The reverse velocity field was insignificant in patterns $C$ and $D$ due to the established pressure gradient that was favorable for directdownstream flow. The vertical velocity component was mostly downward in patterns $A$ and $B$, whereas in patterns $C$ and $D$ the vertical velocity component was mostly upward below the step height. The vertical velocity component was generally diminished further downstream, and the flow pattern approached that of a typical channel flow.

The fluid injected was observed to flow into the shear layer near the tip of backstep and the level of Reynolds stress there was decreased accordingly. The decreasing effect was limited within the region near the streamwise stage $x=0.15 X_{\mathrm{r}}$ under pattern $\mathrm{B}$, whereas the region affected extended toward the streamwise stage $x=0.45 X_{\mathrm{r}}$ in patterns $\mathrm{C}$ and D. Similar trend was also revealed in the distribution of turbulent kinetic energy.

\section{References}

[1] J.K. Eaton, J.P. Johnston, A review of research on subsonic turbulent flow reattachment, AIAA J. 19 (1981) 1093-1100.

[2] P. Bradshaw, F.Y.F. Wong, The reattachment and relaxation of a turbulent shear layer, J. Fluid Mech. 52 (1972) 113-135.

[3] Y.C. Chao, W.F. Chou, SIMPLER-based procedure for numerical simulation of dynamic vortical characteristics of some turbulent flows, Numer. Heat Transfer B 33 (1998) 37-64.

[4] F. Durst, J.C.F. Pereira, Time-dependent laminar backward-facing step flow in a two-dimensional duct, J. Fluids Eng. 110 (1988) 289-296.

[5] H. Le, P. Moin, J. Kim, Direct numerical simulation of turbulent flow over a backward-facing step, J. Fluid Mech. 330 (1997) 349-374.

[6] P. Koutmos, C. Mavridis, A computational investigation of unsteady separated flows, Int. J. Heat Fluid Flow 18 (1997) 297-306.

[7] J. Richardson, W.A. de Groot, J.I. Jagoda, R.E. Waiterick, J.E. Hubbartt, E.C. Strahle, Solid fuel ramjet simulators results: experimental and analysis in cold flow, J. Propul. Power 24 (1985) 1956-1963.

[8] J.T. Yang, B.B. Tsai, G.L. Tsai, Separated-reattaching flow over a backstep with uniform normal mass bleed, J. Fluids Eng. 116 (1994) 29-35.

[9] Y.T. Yang, C.L. Kuo, Numerical study of a backward-facing step with uniform normal mass bleed, Int. J. Heat Mass Transfer 40 (1997) 1677-1686.

[10] J.T. Yang, J.D. Ku, W.J. Ma, Transient cooling effect by wall mass injection after a backstep in high temperature flow field, Int. J. Heat Mass Transfer 44 (2001) 843-855.

[11] W.J. Ma, Transpiration cooling phenomena in separated flow field, Ph.D. Thesis, National Tsing Hua University, Taiwan, 2000.

[12] T.U. Harinaldi, M. Mizomoto, Effect of slot gas injection to the flow field and coherent structure characteristics of a backstep flow, Int. J. Heat Mass Transfer 44 (2001) 2711-2726.

[13] V. de Brederode, P. Bradshaw, Three-dimensional flow in normally twodimensional separation bubbles: I. Flow behind a rearward-facing step, Imperial College Aeronautics Report 72-19, 1972.

[14] R.W. Pitz, W. Dailey, Combustion in a turbulent mixing layer formed at a rearward-facing step, AIAA J. 21 (1983) 1565-1570. 